Schilp, J., Kruizenga, H.M., Wijnhoven, H.A.H., Leistra, E., Evers, A.M., Binsbergen, J.J. van, Deeg, D.J.H., Visser, M. High prevalence of undernutrition in Dutch community-dwelling older individuals. Nutrition: 2012, 28(11-12), 1151-1156

\begin{tabular}{|l|l|}
$\begin{array}{l}\text { Postprint } \\
\text { Version }\end{array}$ & 1.0 \\
\hline Journal website & http://www.sciencedirect.com/science/article/pii/S0899900712000949 \\
\hline Pubmed link & $\underline{\text { http://www.ncbi.nlm.nih.gov/pubmed/22749873 }}$ \\
\hline DOI & $10.1016 /$ j.nut.2012.02.016
\end{tabular}

This is a NIVEL certified Post Print, more info at http://www.nivel.eu

\title{
High prevalence of undernutrition in Dutch community-dwelling older individuals
}

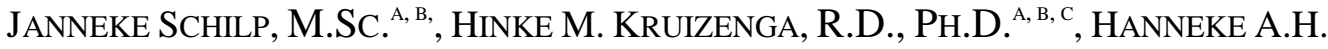 \\ WiJnhOVEn, Ph.D. ${ }^{A}$, Eva Leistra, M.SC. ${ }^{\mathrm{B}, \mathrm{C}}$, ANJA M. EVERS, R.D., M.SC. ${ }^{\mathrm{B}}$, JAAP J. VAN \\ Binsbergen, M.D., Ph.D. ${ }^{\mathrm{B}, \mathrm{D}}$, Dorly J.H. DeEg, Ph.D. ${ }^{\mathrm{E}}$, MARJolein Visser, Ph.D. ${ }^{\mathrm{A}, \mathrm{B}, \mathrm{E}}$ \\ ${ }^{a}$ Department of Health Sciences and the EMGO Institute for Health and Care Research, \\ Faculty of Earth and Life Sciences, VU University, Amsterdam, The Netherlands \\ ${ }^{b}$ Dutch Malnutrition Steering Group, Amsterdam, The Netherlands \\ ${ }^{c}$ Department of Nutrition and Dietetics, Internal Medicine, and the EMGO Institute for Health \\ and Care Research, VU University Medical Center, Amsterdam, The Netherlands \\ ${ }^{d}$ Department Primary and Community Care, Radboud University Nijmegen Medical Centre, \\ Nijmegen, The Netherlands \\ ${ }^{\mathrm{e}}$ Department of Epidemiology and Biostatistics and the EMGO Institute for Health and Care \\ Research, VU University Medical Center, Amsterdam, The Netherlands
}

\begin{abstract}
Objective

To examine the prevalence of undernutrition in community-dwelling older individuals ( $\geq 65 \mathrm{y}$ ) using data from various settings.

Methods

A cross-sectional observational study was performed to examine the prevalence of undernutrition in three samples (all $\geq 65 \mathrm{y}$ ): 1) 1267 community-dwelling individuals participating in a large prospective population-based study, the Longitudinal Aging Study Amsterdam (LASA) in 1998/99; 2) 814 patients receiving home care in 2009/10; and 3) 1878 patients from general practices during the annual influenza vaccination in 2009/10. Undernutrition was assessed by the Short Nutritional Assessment Questionnaire 65+.

Results

Mean age was 77.3 y (SD 6.7) in the LASA sample, 81.6 y (SD 7.4) in the home care sample, and 75.3 y (SD 6.5) in the general practice sample. The prevalence of undernutrition was highest in the home care sample (35\%), followed by the general practice (12\%) and LASA (11\%) samples. The prevalence of undernutrition increased significantly with age in the general practice and LASA samples. Gender differences were observed in the general practice and home care samples; women were more likely to be undernourished in the general
\end{abstract}


Schilp, J., Kruizenga, H.M., Wijnhoven, H.A.H., Leistra, E., Evers, A.M., Binsbergen, J.J. van, Deeg, D.J.H., Visser, M. High prevalence of undernutrition in Dutch community-dwelling older individuals. Nutrition: 2012, 28(11-12), 1151-1156

practice sample and men were more likely to be undernourished in the home care sample.

Conclusion

The prevalence of undernutrition in Dutch community-dwelling older individuals was relatively high, especially in home care patients.

Keywords

Undernutrition;

Aged;

Independently living;

Home care;

General practice

\section{INTRODUCTION}

Undernutrition is an important problem in all health care settings. Undernutrition can be defined as a disorder of nutritional status resulting from reduced nutrient intake or impaired metabolism [1]. In Western society, the presence of undernutrition is found to be associated with delayed wound healing [2] and [3], impaired immune function [4], poor muscle function [5] and [6], mental health problems [7] and [8], impaired quality of life [9] and [10], and even increased morbidity and mortality rates [11], [12], [13], [14] and [15]. In the Netherlands in 2010, the prevalence of undernutrition was estimated to be $25 \%$ in hospitals, $21 \%$ in nursing home residents, and $17 \%$ in patients receiving home care [16]. Although undernutrition is present in all age groups, the prevalence of undernutrition increases with age [16], [17] and [18] and appears to be highest in older individuals [15], [19], [20] and [21]. Studies performed in institutionalized older patients showed that treatment of undernutrition could lead to improved wound healing [22] and [23], less complications [24], better quality of life [24] and [25], and lower mortality [26].

In the past years, more attention is given to recognize and treat undernourished patients in Dutch institutional settings. Screening and treatment of undernutrition in hospital patients were added as performance indicators to the national benchmarks on quality of care in the Netherlands in 2007/08 [27]. On the contrary, recognition and treatment of undernutrition in older individuals in the home situation has received less attention. The results of the Dutch Annual National Prevalence Measurement of Care Problems (LPZ Prevalence Study) in 2010 showed that nutritional status was assessed in only $16 \%$ of the home care patients [16]. In $71 \%$ of this subgroup, undernutrition was assessed by just looking at the patient and in only $5 \%$ a validated screening instrument was used [16]. The Dutch College of General Practitioners introduced the "National Primary Care Cooperation Agreement Undernutrition" on the collaboration of primary care workers in 2010 to enhance awareness and early intervention in the case of undernutrition [28]. Recognition of undernutrition in an early phase is important to initiate timely treatment and to prevent aggravation of the nutritional status. The importance of early detection is emphasized by the fact that older individuals have a reduced ability to recover from weight loss [29].

Studies determining the prevalence of undernutrition in community-dwelling older individuals are scarce. Depending on the specific older study population and the definition used to determine undernutrition, prevalences range from 0 to $24 \%$ [16], [30] and [31]. More knowledge about the prevalence in the specific populations at 
Schilp, J., Kruizenga, H.M., Wijnhoven, H.A.H., Leistra, E., Evers, A.M., Binsbergen, J.J. van, Deeg, D.J.H., Visser, M. High prevalence of undernutrition in Dutch community-dwelling older individuals. Nutrition: 2012, 28(11-12), 1151-1156

risk of undernutrition in the home situation is needed to provide recommendations for the assessment and treatment of undernutrition in community-dwelling older individuals. Recently, a new instrument was specifically developed and validated to determine undernutrition in community-dwelling older individuals: the Short Nutritional Assessment Questionnaire 65+ $\left(\mathrm{SNAQ}^{65+}\right)$ [32]. This instrument is feasible and fast to use, without the need of any calculation or heavy equipment, and is therefore well applicable in the home situation. The aim of the present study was to identify the prevalence of undernutrition in three different samples of Dutch community-dwelling older individuals using the $\mathrm{SNAQ}^{65+}$.

\section{Materials and methods}

Data of three samples were collected within two cohort studies: one sample from the Longitudinal Aging Study Amsterdam (LASA) and two samples from the Nutrition in Primary Care Study (NPCS). Both studies were approved by the Ethics Review Board of the VU University Medical Center and informed consent was obtained from all participants.

\section{Study samples}

LASA is an ongoing cohort study focusing on predictors and consequences of changes in autonomy and well-being in the aging population in the Netherlands. A representative sample of older individuals (55 to $85 \mathrm{y}$ old), stratified by age and sex according to expected mortality after $5 \mathrm{y}$, was drawn from the population registries of 11 municipalities in areas in the west (Amsterdam and vicinity), northeast (Zwolle and vicinity), and south (Oss and vicinity) of the Netherlands. Further details about the sampling and data-collection procedures have been described elsewhere [33]. A total of 3107 participants were enrolled at the baseline examination (1992/1993). Examinations were performed every 3 y and consist of a general face-to-face interview and a medical interview at the participant's home. Data for the present study were collected in 1998 and 1999, in a medical interview by trained research nurses using a standardized protocol. Participants aged $\geq 65$ y $(n=1289)$ were included. Subsequently, participants with missing data on nutritional status were excluded $(n=22)$, resulting in a sample of 1267 participants.

NPCS is an ongoing intervention study investigating the (cost) effectiveness of early treatment by a dietitian of undernourished community-dwelling older individuals in Dutch primary care and home care. Undernourished participants were recruited through 12 general practices and a home care organization in Amsterdam and vicinity. Nutritional status was assessed by 24 research assistants during the annual influenza vaccination on a specific day in the general practices from October 2009 to December 2009 (eight general practices) or in November 2010 (four general practices). After exclusion of individuals with missing data on gender $(n=25), 1878$ participants aged $\geq 65 \mathrm{y}$ were included in the sample. In the home care organization, nurses were trained to assess nutritional status at the individual's home during an intake consultation when the care needs were determined or during an evaluation consultation. Terminally ill individuals or individuals suffering from dementia were excluded from the assessment. Data collected by 54 home care nurses between November 2009 and December 2010 were used. Individuals with missing data on gender $(n=1)$ or nutritional status $(n=18)$ were excluded, resulting in a sample of 814 participants aged $\geq 65 \mathrm{y}$. 
Schilp, J., Kruizenga, H.M., Wijnhoven, H.A.H., Leistra, E., Evers, A.M., Binsbergen, J.J. van, Deeg, D.J.H., Visser, M. High prevalence of undernutrition in Dutch community-dwelling older individuals. Nutrition: 2012, 28(11-12), 1151-1156

The total study sample consisted of 1267 participants from the LASA study, 1878 participants from the general practices, and 814 participants receiving home care.

\section{Nutritional status}

Undernutrition was assessed by the $\mathrm{SNAQ}^{65+}[32]$. This instrument consists of four items: the measurement of mid-upper arm circumference (MUAC) and three questions on involuntary weight loss in the past 6 mo, poor appetite, and difficulties walking stairs. Participants with a MUAC $<25 \mathrm{~cm}$ and/or involuntary weight loss $\geq 4$ $\mathrm{kg}$ in the past 6 mo were defined as undernourished. Not undernourished participants reporting a poor appetite in the past week in combination with reporting difficulties climbing stairs were defined as being at risk of undernutrition [32]. In LASA the answers on the items were defined retrospectively, because the data were already collected.

\section{Weight loss}

To determine involuntary weight loss in the past 6 mo in the LASA sample, the answers on three questions were used: 1) "did your weight change in the past 6 months?”; 2) "how many kilograms did your weight change?”; and 3) "what is the reason your weight change?” Involuntary weight loss was defined as weight loss due to disease, poor appetite, or social factors, or a by the participant reporting "unknown" reason. A cutoff point of $\geq 4 \mathrm{~kg}$ involuntary weight loss in the past 6 mo was used to define undernutrition. This corresponds with a $5 \%$ weight change in the LASA study [32]. In the NPCS samples, one question was asked to define involuntarily weight loss: "Did you involuntary lose 4 kilogram or more in the past six months?” with answering categories yes and no.

\section{Mid-upper arm circumference}

MUAC was measured at the left arm to the nearest millimeter at a point midway between the lateral projection of the acromion process of the scapula and the inferior margin of the olecranon process of the ulna. The midway point was determined with the arm bent at the elbow at a 90-degree angle, while the actual measure was performed with the arm hanging loose. In LASA, the MUAC was measured in duplicate, whereby the mean of two MUAC measurements was used in the analyses. MUAC was dichotomized into $<25 \mathrm{~cm}$ and $\geq 25 \mathrm{~cm}$ based on the 5 th percentile of the total LASA study sample [34].

\section{Appetite}

In the LASA sample, appetite during the past week was assessed with the following question from the Dutch translation of the Center for Epidemiologic Studies Depression Scale: "In the past week, I did not feel like eating; my appetite was poor" [35]. Two categories were created: no problems with appetite (answer rarely or never) and poor appetite last week (answer some of the time/occasionally/mostly or always). In the NPCS samples appetite was assessed by the question: "Did you have a poor appetite in the past week?”, with answering categories yes and no.

\section{Walking stairs}

Difficulty walking up and down a staircase was assessed by the question "Can you walk up and down a staircase of 15 steps without resting?” In the LASA sample, two categories were created: no difficulties (answer yes, without help) and difficulties (answer yes, with some/much difficulty/only with help/no, I cannot). In the NPCS samples response categories were yes and no. 
Schilp, J., Kruizenga, H.M., Wijnhoven, H.A.H., Leistra, E., Evers, A.M., Binsbergen, J.J. van, Deeg, D.J.H., Visser, M. High prevalence of undernutrition in Dutch community-dwelling older individuals. Nutrition: 2012, 28(11-12), 1151-1156

\section{Statistical analyses}

The prevalence of (the risk of) undernutrition with the SNAQ ${ }^{65+}$ was calculated in the three different study samples and characteristics of the study samples were examined. Differences between the study samples were tested using ANOVA for continuous variables and $\chi^{2}$ tests for dichotomous and categorical variables. The percentage of undernourished participants with a MUAC $<25 \mathrm{~cm}$, with $\geq 4 \mathrm{~kg}$ involuntary weight loss in the past 6 mo or both, were calculated for every sample. The prevalence of undernutrition was presented in age quintiles (based on including all three individual samples) and for men and women separately. Differences were tested with $\chi^{2}$ test and linear-by-linear associations were calculated to obtain insight into the trend of the prevalence across age quintiles. A $P$ value $<0.05$ was considered statistically significant. The analyses were performed using SPSS version 16.0 (SPSS Inc., Chicago, IL, USA).

\section{RESULTS}

Table 1 shows the characteristics of the three study samples and the prevalence of (the risk of) undernutrition. In total, 3959 participants (59.2\% women) were included in the study, with a mean age of 77.2 (SD 7.2) y. The home care sample differed from the other samples on all investigated characteristics. Participants in the home care sample were more often women, were older, and had the lowest mean MUAC $(P<0.001)$. The characteristics of the LASA and general practice samples were most comparable. The prevalence of undernutrition was $10.7 \%$ (95\% CI 9.0, 12.4) in the LASA sample, $11.8 \%$ (95\% CI 10.3, 13.3) in the general practice, and 34.8\% (95\% CI 31.5, 38.1) in the home care sample. The risk of undernutrition was 7.7\% (95\% CI $6.2,9.2)$ in the LASA sample, $2.2 \%$ (95\% CI 1.4, 3.0) in the general practice sample, and $9.2 \%(95 \%$ CI $7.6,10.8)$ in the home care sample. The mean overall prevalence of undernutrition was $16.2 \%(95 \%$ CI $15.0,17.4)$ and the mean overall prevalence of the risk of undernutrition was 5.4\% (95\% CI 4.1, 6.7). Additional characteristics of the LASA sample were examined: $12 \%$ had a poor cognitive status (Mini-Mental State Examination score $\leq 23$ ), $39 \%$ had a poor self-perceived health, and $88 \%$ reported having one or more chronic diseases. The mean handgrip strength of the LASA sample was $31.9 \mathrm{~kg}$ (SD 9.7) in men and $18.9 \mathrm{~kg}$ (SD 6.9) in women. Furthermore, $70 \%$ of the men and $34 \%$ of the women were married and $22 \%$ of the men and $54 \%$ of the women were widowed. No comparison on these characteristics could be made between the samples, as this information was not available for the other two study samples.

\section{[TABLE 1]}

The underlying criteria for undernutrition according to the SNAQ ${ }^{65+}$ are illustrated in Figure 1. In the LASA and general practice samples most undernourished participants were undernourished based on a low MUAC. In LASA a statistically significant difference was found between men and women $(P=0.04)$. In the home care sample most undernourished participants were undernourished based on their involuntary weight loss $\geq 4 \mathrm{~kg}$. This percentage was significantly higher in men compared to women $(P=0.003)$. In the home care sample almost one of four undernourished participants was undernourished based on both criteria. 
Schilp, J., Kruizenga, H.M., Wijnhoven, H.A.H., Leistra, E., Evers, A.M., Binsbergen, J.J. van, Deeg, D.J.H., Visser, M. High prevalence of undernutrition in Dutch community-dwelling older individuals. Nutrition: 2012, 28(11-12), 1151-1156

\section{[FIGURE 1]}

Figure 2 shows the prevalence of undernutrition for the age quintiles in the total study sample. The prevalence of undernutrition increased statistically significantly ( $P$ $<0.001$ ) with age in the general practice and LASA samples. In these samples the prevalence was highest in the age group $\geq 85 \mathrm{y} ; 20.9 \%$ (95\% CI 15.2, 26.6) in the LASA sample, and $22.8 \%(95 \%$ CI 16.3, 29.3) in the general practice sample. In the home care sample, the prevalence of undernutrition did not differ between the age quintiles.

\section{[FIGURE 2]}

The prevalence of the risk of undernutrition differed significantly between the age quintiles in the LASA sample, but there was no trend across the age quintiles (linearby-linear association, $P=0.46)$. The highest prevalence $(13.0 \%$; 95\% CI 10.4, 15.6) was found in the age group $80-84 \mathrm{y}$ and the lowest prevalence $(4.9 \%$; $95 \%$ CI 3.3, 6.5 ) was found in the age group $\geq 85 \mathrm{y}$. In the general practice and home care samples no statistically significant differences were found between the age quintiles. In the home care sample, the prevalence ranges from $5.9 \%$ (95\% CI 4.1, 7.7) in the age group $65-69$ y to $11.1 \%(95 \%$ CI $8.7,13.5)$ in the age group $\geq 85 \mathrm{y}$. In the general practice sample, the prevalence ranges from $1.0 \%(95 \%$ CI $0.2,1.8)$ in the age group $70-74$ y to $3.0 \%(95 \%$ CI $1.7,4.3)$ in the age group $\geq 85 \mathrm{y}$.

Figure 3 shows the prevalence of (the risk of) undernutrition for men and women in the three study samples. In the general practice and home care samples statistically significant differences were found between men and women. Women were more likely to be undernourished than men in the general practice sample $(P<0.001)$. In the home care sample men were more likely to be undernourished than women $(P=$ 0.02). In the LASA sample no significant gender differences were found. An additional analysis showed that potential age differences between men and women did not explain the observed gender differences in prevalence.

\section{[FIGURE 3]}

\section{DisCussion}

In Dutch community-dwelling older individuals $(\geq 65 \mathrm{y})$, the prevalence of undernutrition was $11 \%$ in a representative sample of 1267 community-dwelling older individuals from the LASA study, $12 \%$ in a sample of 1878 general practice patients (during the annual influenza vaccination), and 35\% in a sample of 814 home care patients (during an intake or evaluation consultation). The prevalence of undernutrition increased statistically significantly with age in the LASA and general practice samples and gender differences were observed in the general practice and home care samples.

This is the first study investigating the prevalence of undernutrition in communitydwelling older individuals using the SNAQ ${ }^{65+}$. Thereby, comparing the observed prevalences to the results of other studies is difficult, because they largely depend on the used criteria to define (the risk of) undernutrition and the considered population and setting. Studies reporting the prevalence in older individuals in general practice are scarce, with values ranging from $0 \%$ assessed with the Mini Nutritional Assessment [36] to $11.6 \%$ using a low body mass index (BMI) as the criteria (the used cutoff point for low BMI was not reported) [37]. The prevalence of 
Schilp, J., Kruizenga, H.M., Wijnhoven, H.A.H., Leistra, E., Evers, A.M., Binsbergen, J.J. van, Deeg, D.J.H., Visser, M. High prevalence of undernutrition in Dutch community-dwelling older individuals. Nutrition: 2012, 28(11-12), 1151-1156

undernutrition observed in our general practice and LASA samples is comparable to the latter study. The prevalence of undernutrition in our home care sample is higher than the prevalence $(17.1 \%)$ found in the earlier mentioned LPZ prevalence study [16]. However, the home care sample of the LPZ prevalence study was younger (mean age $76.2 \mathrm{y}$ ) compared to our sample (mean age $81.8 \mathrm{y}$ ). In addition, more stringent criteria were used to assess undernutrition in the LPZ prevalence study: $\mathrm{BMI} \leq 20 \mathrm{~kg} / \mathrm{m}^{2},>6 \mathrm{~kg}$ involuntary weight loss in the past 6 mo or $>3 \mathrm{~kg}$ in the past month, and reduced nutritional intake. The cutoff value of $25 \mathrm{~cm}$ for MUAC used in our study was comparable with a BMI of $20.7 \mathrm{~kg} / \mathrm{m}^{2}$ in LASA (approximated with a linear regression analysis). Moreover, the cutoff value for involuntary weight loss ( $\geq 4 \mathrm{~kg}$ ) was also less strict in our study compared to the LPZ prevalence study. The increasing prevalence of undernutrition with age shown in earlier studies [17], [18], [20] and [21] was confirmed in the LASA and general practice samples, but not in the home care sample. Besides the increasing prevalence of undernutrition, other health problems and diseases such as depression, cancer, heart disease, and the presence of multimorbidities are also known to increase with increasing age [38], [39], [40], [41] and [42]. The decreasing prevalence in the home care sample was comparable to the results of the LPZ prevalence study and could be due to the assumption that older individuals with higher disease severity are more likely to die or to be admitted to an institution, whereby the healthier older individuals will be more likely to stay at home [16]. Because of the observed age differences in the general practice sample, it could be useful to consider only assessing undernutrition in the highest age groups in this setting.

The contradictory results between the samples with regard to gender differences in the prevalence of undernutrition are difficult to interpret. In general, women are more often frail than men [43], which was reflected in the prevalence of undernutrition in our general practice sample. The higher prevalence of undernutrition in men compared to women in our home care sample could be due to the fact that the frailest patients in home care are more likely to be men [44]. Women generally receive home care more often compared to men, because women are more often living without a partner, but men are more frail [45]. An earlier study pooled data from published data sets and showed that the prevalence of undernutrition was higher in communitydwelling older men compared to women [46], but it was not mentioned whether this population received home care. In the LPZ prevalence study no statistically significant gender differences were found in the home care setting [47]. Based on the results of our study, we will recommend assessing nutritional status in both men and women and not differentiating the assessment for gender. One strength of our study is that three large and diverse samples were used to determine the prevalence of undernutrition in community-dwelling older individuals. Probably some overlap exists between the three samples, because, for example, individuals assessed during the influenza vaccination in general practices as well as participants of LASA could also potentially receive home care. Another strength is the unique direct comparison of different settings of community-dwelling older individuals. Advantage of assessment during the influenza vaccination in general practice or during consultation in home care is that assessment can be performed regularly in large samples of older individuals, allowing monitoring of nutritional status over time. 
Schilp, J., Kruizenga, H.M., Wijnhoven, H.A.H., Leistra, E., Evers, A.M., Binsbergen, J.J. van, Deeg, D.J.H., Visser, M. High prevalence of undernutrition in Dutch community-dwelling older individuals. Nutrition: 2012, 28(11-12), 1151-1156

A limitation of this study is that undernutrition in the LASA sample was assessed in 1998/99, while undernutrition in the other two samples was assessed in 2009/10. The MUAC was only measured until the third cycle of LASA (1998/99) and more recent cycles could therefore not be used to determine the prevalence of undernutrition based on the SNAQ ${ }^{65+}$. An additional analysis, using BMI $<20 \mathrm{~kg} / \mathrm{m}^{2}$ instead of MUAC $<25 \mathrm{~cm}$, showed comparable prevalences of undernutrition between 1998/99 (6.3\%) and 2005/06 (6.6\%) in individuals between age 65 and 85 y. These data suggest that the prevalences did not vary over time, allowing a direct comparison of the three study samples. Another potential limitation was that the questions used in the SNAQ ${ }^{65+}$ were not asked identically in the LASA sample as compared to the other two samples, which may explain some of the differences in the prevalence between the samples.

In the present study home care nurses were instructed to assess the nutritional status of all individuals aged $65 \mathrm{y}$ and older during an intake or evaluation consultation, but not all individuals were actually assessed because terminally ill individuals or individuals suffering from dementia were excluded from assessment, causing selection bias. Furthermore, it is possible that during the start-up phase nurses may have selectively screened those individuals who appeared undernourished. However, the prevalence of undernutrition (38.1\%; 95\% CI 33.2, 43.0) of the first 4 mo (November 2009 to February 2010) was not statistically significantly different $(P=$ $0.16)$ from the prevalence (31.7\%; 95\% CI 27.0, 36.4) of the last 4 mo of recruitment (September to December 2010).

This study demonstrates that the prevalence of undernutrition in community-dwelling older individuals is substantial. The prevalence of undernutrition was highest in a sample of older individuals receiving home care, in both men and women and in all age groups ( $\geq 65 \mathrm{y}$ ). Therefore, assessment of undernutrition in home care during regular consultations is warranted. In general practice, almost one of four patients (both men and women) aged $85 \mathrm{y}$ and older was undernourished during the influenza vaccination. Concerning investment of time and money, it could be useful to consider only assessing undernutrition in the highest age groups in general practice. Early recognition of undernutrition in community-dwelling older individuals is important to initiate treatment in a timely fashion and prevent further deterioration of nutritional status.

ACKNOWLEDGMENTS

LASA and NPCS were largely funded by the Ministry of Health, Welfare, and Sports of the Netherlands.

\section{REFERENCES}

[1]D.A. August, R.F. KushnerThe 1995 ASPEN standards for nutrition support: hospitalized patientsNutr Clin Pract, 10 (1995), pp. 206-207

[2]D.A. Haydock, G.L. Hilllmpaired wound healing in surgical patients with varying degrees of malnutritionJ Parenter Enteral Nutr, 10 (1986), pp. 550-554

[3]E.S. Shahin, J.M. Meijers, J.M. Schols, A. Tannen, R.J. Halfens, T. DassenThe relationship between malnutrition parameters and pressure ulcers in hospitals and nursing homesNutrition, 26 (2010), pp. 886-889

[4]K. Kawakami, J. Kadota, K. lida, R. Shirai, K. Abe, S. KohnoReduced immune function and malnutrition in the elderlyTohoku J Exp Med, 187 (1999), pp. 157-171 
Schilp, J., Kruizenga, H.M., Wijnhoven, H.A.H., Leistra, E., Evers, A.M., Binsbergen, J.J. van, Deeg, D.J.H., Visser, M. High prevalence of undernutrition in Dutch community-dwelling older individuals. Nutrition: 2012, 28(11-12), 1151-1156

[5]I. Bourdel-Marchasson, P.A. Joseph, P. Dehail, M. Biran, P. Faux, M. Rainfray et al.Functional and metabolic early changes in calf muscle occurring during nutritional repletion in malnourished elderly patientsAm J Clin Nutr, 73 (2001), pp. 832-838

[6]M.P. Engelen, A.M. Schols, W.C. Baken, G.J. Wesseling, E.F. WoutersNutritional depletion in relation to respiratory and peripheral skeletal muscle function in out-patients with COPDEur Respir J, 7 (1994), pp. 1793-1797

[7]M.A. Cabrera, A.E. Mesas, A.R. Garcia, S.M. de AndradeMalnutrition and depression among community-dwelling elderly peopleJ Am Med Dir Assoc, 8 (2007), pp. 582-584

[8]J.M. Kvamme, O. Gronli, J. Florholmen, B.K. JacobsenRisk of malnutrition is associated with mental health symptoms in community living elderly men and women: the Tromso studyBMC Psychiatry, 11 (2011), p. 112

[9]E. Amarantos, A. Martinez, J. DwyerNutrition and quality of life in older adultsJ Gerontol A Biol Sci Med Sci, 56 (Spec No 2) (2001), pp. 54-64

[10]D.R. ThomasThe critical link between health-related quality of life and age-related changes in physical activity and nutritionJ Gerontol A Biol Sci Med Sci, 56 (2001), pp. 599 602

[11]M.M. Corrada, C.H. Kawas, F. Mozaffar, A. Paganini-HillAssociation of body mass index and weight change with all-cause mortality in the elderlyAm J Epidemiol, 163 (2006), pp. 938-949

[12]L. Liu, M.M. Bopp, P.K. Roberson, D.H. SullivanUndernutrition and risk of mortality in elderly patients within 1 year of hospital dischargeJ Gerontol A Biol Sci Med Sci, 57 (2002), pp. 741-746

[13]J.L. Locher, D.L. Roth, C.S. Ritchie, K. Cox, P. Sawyer, E.V. Bodner et al.Body mass index, weight loss, and mortality in community-dwelling older adultsJ Gerontol A Biol Sci Med Sci, 62 (2007), pp. 1389-1392

[14]D.H. Sullivan, M.M. Bopp, P.K. RobersonProtein-energy undernutrition and lifethreatening complications among the hospitalized elderlyJ Gen Intern Med, 17 (2002), pp. 923-932

[15]L.S. Ferreira, T.F. do Amaral, M. de Fatima Nunes Marucci, L.F. Costa Nascimento, M.L. Lebrao, Y.A. de Oliveira DuarteUndernutrition as a major risk factor for death among older Brazilian adults in the community-dwelling setting: SABE surveyNutrition, 27 (2011), pp. 1017-1022

[16]R.J.G. Halfens, J.M.M. Meijers, M.F.M.T. Du Moulin, N.C. Van Nie, J.C.L. Neyers, J.M.G.A. ScholsRapportage resultaten: Landelijke Prevalentiemeting Zorgproblemen (National Prevalence Measurement of Care Problems)Maastricht University, Maastricht, The Netherlands (2010)

[17]M. Pirlich, T. Schutz, M. Kemps, N. Luhman, G.R. Burmester, G. Baumann et al.Prevalence of malnutrition in hospitalized medical patients: impact of underlying diseaseDig Dis, 21 (2003), pp. 245-251

[18]A.B. Newman, D. Yanez, T. Harris, A. Duxbury, P.L. Enright, L.P. FriedWeight change in old age and its association with mortalityJ Am Geriatr Soc, 49 (2001), pp. 1309-1318

[19]B.M. Margetts, R.L. Thompson, M. Elia, A.A. JacksonPrevalence of risk of undernutrition is associated with poor health status in older people in the UKEur J Clin Nutr, 57 (2003), pp. $69-74$

[20]Y. Johansson, M. Bachrach-Lindstrom, J. Carstensen, A.C. EkMalnutrition in a homeliving older population: prevalence, incidence and risk factors. A prospective studyJ Clin Nurs, 18 (2009), pp. 1354-1364

[21]E. Perissinotto, C. Pisent, G. Sergi, F. GrigolettoAnthropometric measurements in the elderly: age and gender differencesBr J Nutr, 87 (2002), pp. 177-186

[22]A.C. Ek, M. Unosson, J. Larsson, H. Von Schenck, P. BjurulfThe development and healing of pressure sores related to the nutritional stateClin Nutr, 10 (1991), pp. 245-250

[23]C.E. Collins, J. Kershaw, S. BrockingtonEffect of nutritional supplements on wound healing in home-nursed elderly: a randomized trialNutrition, 21 (2005), pp. 147-155

[24]J. Starke, H. Schneider, B. Alteheld, P. Stehle, R. MeierShort-term individual nutritional care as part of routine clinical setting improves outcome and quality of life in malnourished medical patientsClin Nutr, 30 (2011), pp. 194-201 
Schilp, J., Kruizenga, H.M., Wijnhoven, H.A.H., Leistra, E., Evers, A.M., Binsbergen, J.J. van, Deeg, D.J.H., Visser, M. High prevalence of undernutrition in Dutch community-dwelling older individuals. Nutrition: 2012, 28(11-12), 1151-1156

[25]U. Rufenacht, M. Ruhlin, M. Wegmann, R. Imoberdorf, P.E. BallmerNutritional counseling improves quality of life and nutrient intake in hospitalized undernourished patientsNutrition, 26 (2010), pp. 53-60

[26]A.C. Milne, J. Potter, A. Vivanti, A. AvenellProtein and energy supplementation in elderly people at risk from malnutritionCochrane Database Syst Rev (2009), p. CD003288

[27]Dutch Health Care Inspectorate. Performance indicators hospitals, 2010. Utrecht: Dutch Health Care Inspectorate; 2009.

[28]M.J.P. Van Avendonk, P. Mensink, T. Drenthen, J.J. Van BinsbergenPrimary care and publich health a natural alliance? The introduction of the guidelines for obesity and undernutrition of the Dutch College of General PractitionersFam Practice, 29 (2012), pp. i31-i35

[29]S.B. Roberts, P. Fuss, M.B. Heyman, W.J. Evans, R. Tsay, H. Rasmussen et al.Control of food intake in older menJAMA, 272 (1994), pp. 1601-1606

[30]C.C. Chen, L.S. Schilling, C.H. LyderA concept analysis of malnutrition in the elderlyJ Adv Nurs, 36 (2001), pp. 131-142

[31]C.A. Van Wayenburg, F.A. Van de Laar, C. Van Weel, W.A. Van Staveren, J.J. Van BinsbergenNutritional deficiency in general practice: a systematic reviewEur J Clin Nutr, 59 (Suppl 1) (2005), pp. S81-S87 discussion S88

[32]H.A. Wijnhoven, J. Schilp, M.A. Van Bokhorst-de van der Schueren, H.C. De Vet, H.M. Kruizenga, D.J.H. Deeg et al.Development and validation of criteria for determining undernutrition in community-dwelling older men and women: the Short Nutritional Assessment Questionnaire 65+Clin Nutr, 31 (2012), pp. 351-358

[33]M. Huisman, J. Poppelaars, M. van der Horst, A.T. Beekman, J. Brug, T.G. van Tilburg et al.Cohort Profile: The Longitudinal Aging Study AmsterdamInt J Epidemiol, 40 (2011), pp. 868-876

[34]H.A. Wijnhoven, M.A. van Bokhorst-de van der Schueren, M.W. Heymans, H.C. de Vet, H.M. Kruizenga, J.W. Twisk et al.Low mid-upper arm circumference, calf circumference, and body mass index and mortality in older personsJ Gerontol A Biol Sci Med Sci, 65 (2010), pp. 1107-1114

[35]L. RadloffThe CES-D scale: a self-reported depression scale for research in the general populationAppl Psychol Measur, 1 (1977), pp. 385-401

[36]A.M. Beck, L. Ovesen, M. SchrollA six months' prospective follow-up of 65+-y-old patients from general practice classified according to nutritional risk by the Mini Nutritional AssessmentEur J Clin Nutr, 55 (2001), pp. 1028-1033

[37]P.A. DeVoreAssessment of nutritional status and obesity in elderly patients as seen in general medical practiceSouth Med J, 86 (1993), pp. 1008-1010

[38]W.W. Hung, J.S. Ross, K.S. Boockvar, A.L. SiuRecent trends in chronic disease, impairment and disability among older adults in the United StatesBMC Geriatr, 11 (2011), p. 47

[39]C. Salisbury, L. Johnson, S. Purdy, J.M. Valderas, A.A. MontgomeryEpidemiology and impact of multimorbidity in primary care: a retrospective cohort studyBr J Gen Pract, 61 (2011), pp. e12-e21

[40]M.L. Janssen-Heijnen, S. Houterman, V.E. Lemmens, M.W. Louwman, H.A. Maas, J.W. CoeberghPrognostic impact of increasing age and co-morbidity in cancer patients: a population-based approachCrit Rev Oncol Hematol, 55 (2005), pp. 231-240

[41]H.I. Solhaug, E.B. Romuld, U. Romild, E. Stordallncreased prevalence of depression in cohorts of the elderly: an 11-year follow-up in the general population-the HUNT studyint Psychogeriatr, 24 (2012), pp. 151-158

[42]J.W. Davis, R. Chung, D.T. JuarezPrevalence of comorbid conditions with aging among patients with diabetes and cardiovascular diseaseHawaii Med J, 70 (2011), pp. 209-213

[43]M.T. Puts, P. Lips, D.J. DeegSex differences in the risk of frailty for mortality independent of disability and chronic diseasesJ Am Geriatr Soc, 53 (2005), pp. 40-47

[44]J.J. Armstrong, P. Stolee, J.P. Hirdes, J.W. PossExamining three frailty conceptualizations in their ability to predict negative outcomes for home-care clientsAge Ageing, 39 (2010), pp. 755-758

[45]S.W. Geerlings, A.M. Pot, J.W.R. Twisk, D.J.H. DeegPredicting transitions in the use of informal and professional care by older adultsAgeing Soc, 25 (2005), pp. 111-130

View Record in Scopus 
Schilp, J., Kruizenga, H.M., Wijnhoven, H.A.H., Leistra, E., Evers, A.M., Binsbergen, J.J. van, Deeg, D.J.H., Visser, M. High prevalence of undernutrition in Dutch community-dwelling older individuals. Nutrition: 2012, 28(11-12), 1151-1156

[46]M.J. Kaiser, J.M. Bauer, C. Ramsch, W. Uter, Y. Guigoz, T. Cederholm et al.Frequency of malnutrition in older adults: a multinational perspective using the mini nutritional assessmentJ Am Geriatr Soc, 58 (2010), pp. 1734-1738

[47]J.M. Meijers, J.M. Schols, M.A. van Bokhorst-de van der Schueren, T. Dassen, M.A. Janssen, R.J. HalfensMalnutrition prevalence in The Netherlands: results of the annual Dutch national prevalence measurement of care problemsBr J Nutr, 101 (2009), pp. 417423

\section{TABLES AND FIGURES}

\section{[TABLE 1]}

Characteristics of the study samples and prevalence of undernutrition

\begin{tabular}{|c|c|c|c|c|c|c|}
\hline & \multirow{2}{*}{\begin{tabular}{|c|} 
LASA \\
$N=$ \\
1267
\end{tabular}} & \multirow{2}{*}{$\begin{array}{c}\text { GP } \\
N= \\
1878\end{array}$} & \multirow{2}{*}{$\begin{array}{l}\text { HC } \\
N= \\
814 \\
\end{array}$} & \multicolumn{3}{|c|}{$P$ value * } \\
\hline & & & & $\begin{array}{l}\text { LASA- } \\
\text { GP }\end{array}$ & $\begin{array}{l}\text { LASA- } \\
\text { HC }\end{array}$ & $\begin{array}{l}G P- \\
H C\end{array}$ \\
\hline Women, \% & 54.9 & 57.7 & 69.3 & 0.118 & $<0.001$ & $<0.001$ \\
\hline Age in y, mean (SD) & $\begin{array}{l}77.3 \\
(6.7) \\
\end{array}$ & $\begin{array}{l}75.3 \\
(6.5)\end{array}$ & $\begin{array}{l}81.6 \\
(7.4) \\
\end{array}$ & $<0.001$ & $<0.001$ & $<0.001$ \\
\hline $\begin{array}{l}\text { MUAC in } \mathrm{cm} \text {, mean } \\
\text { (SD) }\end{array}$ & \begin{tabular}{|l|}
30.3 \\
$(3.6)$
\end{tabular} & $\begin{array}{l}29.4 \\
(3.4)\end{array}$ & $\begin{array}{l}28.9 \\
(5.5)\end{array}$ & $<0.001$ & $<0.001$ & 0.001 \\
\hline MUAC $<25 \mathrm{~cm}, \%$ & 5.8 & 7.1 & 15.7 & 0.168 & $<0.001$ & $<0.001$ \\
\hline $\begin{array}{l}\geq 4 \mathrm{~kg} \text { involuntary } \\
\text { weight loss, \% }\end{array}$ & 5.4 & 6.7 & 27.0 & 0.125 & $<0.001$ & $<0.001$ \\
\hline $\begin{array}{l}\text { Poor appetite last week, } \\
\%\end{array}$ & 15.9 & 8.9 & 29.4 & $<0.001$ & $<0.001$ & $<0.001$ \\
\hline $\begin{array}{l}\text { Difficulties walking } \\
\text { stairs, \% }\end{array}$ & 38.3 & 17.0 & 59.2 & $<0.001$ & $<0.001$ & $<0.001$ \\
\hline Nutritional status, $\%$ & & & & $<0.001$ & $<0.001$ & $<0.001$ \\
\hline Undernutrition & 10.7 & 11.8 & 34.8 & & & \\
\hline Risk of undernutrition & 7.7 & 2.2 & 9.2 & & & \\
\hline No undernutrition & 81.7 & 86.0 & 56.0 & & & \\
\hline
\end{tabular}

GP, general practice; HC, home care; LASA, Longitudinal Aging Study Amsterdam; MUAC, mid-upper arm circumference

Differences between the three samples were mutually tested with ANOVA and $\chi^{2}$ tests. 